\title{
Design and fabrication of smart ceiling mop with adjustable head
}

\author{
A. Sivakumar ${ }^{1 *}$ S . Karunakaran ${ }^{2 *}$ K. Ramanathan ${ }^{3}$ K. Sanjay ${ }^{3}$ M.V. Rishikesh ${ }^{3}$ \\ Associate Professor, Department of Mechanical Engineering, Kongu Engineering College, \\ Erode, Tamil Nadu ${ }^{1 *}$ \\ Assistant Professor, Department of Computer Technology (U.G), Kongu Engineering \\ College,Erode, Tamil Nadu ${ }^{2 *}$
}

UG Students, Department of Mechanical Engineering, Kongu Engineering College, Erode, Tamil Nadu ${ }^{3}$

E-mail:askmech@kongu.ac.in

\begin{abstract}
The Ceiling cleaning process is one of the tedious tasks ever which involves removing algae, mold, lichen, dust, and moss from the surface. Cleaning of the roof becomes very difficult when the height of the roof is very high also an excess human effort is required which also makes the labor feel discomfort, in addition to muscle pain. By considering all the above-discussed difficulties, we have developed a concept of a semi-automatic ceiling cleaning machine that is capable of cleaning dirt, grime, algae, etc. on the ceiling. Enabling the machine in accessing hard-to-reach areas on the ceilingsand at higher altitudes. To make the controlling of the machine user-friendly an electric drive is incorporated, such that a person with less technical knowledge can access it with high accuracy. The power source for the entire operation is provided with the help of a battery which is internally installed in the setup. As an added advantage, the multi-head system is implanted, using this our setup can be used as a traditional brush head, and by changing the vacuum head, the setup is capable of performing dust collection operations using vacuum pressure. To make a machine which will take care of the safety of persons, save time and also shouldn't damage false ceiling structure. This method of ceiling cleaning can be used in hotels, offices, etc. which will give a more appealing look. This method is suitable for small, medium as well as large-scale applications.
\end{abstract}

Keywords: semi-automatic, traditional brush head, vacuum head

\section{INTRODUCTION}

The manual, chemical, and mechanical cleaning methods cannot meet the needs of cleaning. Manual cleaning is the most widely practiced method nowadays. The ceiling is cleaned using manpower to scrub it with the wire brush. This method is time consuming and tiring. In the Konkan region of India, especially in the states of Kerala, Maharashtra, and Goa the houses usually have tile roofing, corrugated cement sheeting, etc. These tiles/sheets are arranged along a reliable slope. The cemented sheets are usually used for garages, storehouses, and also in industrial roofing. Upon fitting as the years go by, these tiles/sheets are covered with dust, dried algae, tiny shrubs etc., which makes the roofs look dull and old. By carrying out a cleaning operation the roof is kept clean and at the same time is also increases the life expectancy of the tiles/sheets. In a conventional method of cleaning, cleaners have to climb on top of these roofs and carry out the cleaning operation by scrubbing with the help of wire brushes. This method is tiring and at same time it consumes a lot of time. Also, the person carrying out the cleaning operation can severely injure him/ herself, if he/she falls off the roof causing accidents. There are chances of the person getting 
unconscious due to prolonged exposure to heat during hot weather conditions. Cleaning of ceiling surfaces is a challenging task, as the surface is not even and also has a tendency of breaking easily upon mechanical loading. However, a no. of attempts have been made for cleaning the various roof surfaces at present, only a few are dedicated towards household and industrial roof cleaning.

\section{LITERATURE SURVEY}

Potter et al., (2018) proposed mechanisms to control the infestation of spiders in the buildings. It acknowledges equipment called bellow dusters in applying insecticide dust. Many kinds of spiders live around human settlements. Common spiders that build a web to trap their prey include cellar spiders while wolf spiders prey upon flies and they are mostly harmless to humans. A few spiders that could inflict serious harm to humans that are notable on the corners of the buildings include Black Widow Spider and Brown Recluse Spiders. Not everyone is comfortable with cobwebs and multiple spiders roaming their walls. This article provides suggestions such as routine house cleaning to eliminate spiders using a broom or vacuum cleaner.

Louis et al., (1964) proposed to combine a cluster of feathers such that this construction canbe utilized for a variety of purposes that require a separate duster each. With the novel arrangement of both soft feathers and stiff feathers, the life of the feather duster is prolonged. This feather duster was expected to perform tasks of wiping dust and lifting cobwebs. The design allows for the easy removal of dust and cobwebs from the feathers. This object provides increased static electricity properties due to which the duster can attract and hold dust more effectively. This increased static electric power of the duster is due to the rubbing of various kinds of feathers against each other. The unique arrangement of the duster provides flexibility and simultaneously stiffness, therefore, can be used for universal use. The extension of the handle portion allows cleaning of articles and ceilings which are otherwise inaccessible.

Agustin et al., (1997) developed a device for removing cobwebs with a handle, housing electrical storage cells, and a motor and adjustable length of the rod. When the duster touches the cobwebs, the energized motor spins causing cobwebs to stick and tightly wound around the rod. This device effectively disposes of cobwebs. The device is designed for the quick removal of collected cobwebs from the device itself. The device minimizes the disturbances of nearby items and surfaces while cleaning. The device curbs stress on the wrist while using the device. The device has easy access to narrow gaps on shelves and ceilings. The device is comparatively lighter in weight than the conventional vacuum cleaners and therefore, easily portable.

Liu Fangyi et al., (2009) invented a kind of roof ceiling clearing device, the dust of high place cleaning can be collected and unified the processing. The present invention includes a broom and a Telescopic handlebar. The beneficial effect of the present invention is: the dust use that can be used as tops such as cleaning roof ceiling, the dust that is cleaned can directly fall into and connect ash bucket, and unified processing in leak focuses on the ash bag, dust flies everywhere when avoiding cleaning thus, cause the phenomenon of polluting once more, be beneficial to health and environmental sanitation.

Zhang Shede et al., (2010) invented a kind of Broom that comprise broom head and broomstick, described broomstick comprises a handle, middle handle and following handle, described handle, middle handle, and the following handle inside of going up respectively is 
provided with the cavity that can place foreign material, the described handle end of going up is provided with lid, described upward handle and middle handle are for flexibly connecting, and described middle handle and following handle are to flexibly connect. Because the existence of the last handle, middle handle, and following handle, make three cavities of existence in the besom handle, people both can use besom expediently.

Zhao Feng et al., (2011) invented a cleaning ceiling dust device, cleaning ceiling that can be easy and convenient, and dust cannot fall earthward. The present invention includes the hairbrush that the Telescopic handlebar of the clearing apparatus is connected with the upper end, the technical scheme that adopts is: below the hairbrush that described clearing apparatus connects in Telescopic handlebar upper end, an ash cover is housed. In the Telescopic handlebar lower end, a lanyard is arranged. The beneficial effect of the present invention is: during use, can be with actual needs, andthe release handle pole length, owing to being provided with the ash cover, the dirt that sweeps updust directly falls into unified processing the in cover, cannot produce dust and fly upward the problem that pollutes the environment.

Tang Jianfeng et al., (2012) designed a utility model that discloses a multifunctional sweeper which comprises a bowl-shaped dust cover, triangular support, rollers, a support point, dust conveying pipe, dust receiving the bag, a brush, blades, and a rotating shaft of an electromotor, wherein the brush is connected with the blades, the rotating shaft of the electromotor is connected with the brush and theblades to the same shaft, a power line of the electromotor is connected with a handle of a holding rod through a direction rod, and a control switch is arranged on the handle. The height and the telescopic length of the holding rod are adjusted and fixed, the control switch is switched on, the electromotor rotates to drive the blades and the brush to rotate, airflow drives dust to enter into the dust receiving bag through the dust conveying pipe. The multifunctional sweeper can be used as a device for cleaning a roof ceiling, wall faces, the backside of a hung decorative lighting fixture, the top end of a suspension air conditioner, and other devices on high positions, cleaned dust can directly enter into the dust receiving bag through the dust conveying pipe to be disposed of together, and therefore phenomena of dust flying in cleaning and causing secondary pollution are avoided, the purpose of cleaning dead angles and blind angles on the backside of the lighting fixture on the high position is achieved, an operation is simple, labor-saving and convenient, and the multifunctional weeper is beneficial for body health and environmental hygiene.

Liu Zichen et al., (2013) designed a ceiling sweeper that mainly solves the problem of dust scatters when a ceiling is swept. According to the structure of the ceiling sweeper, the ceiling sweeper comprises a broom, a tapered hopper, a hollow rod, a rocker, and a locking handle. The inverted taper hopper is fixed at the top end of the hollow rod, the rocker is arranged in the inner cavity of the hollow rod, the broom is arranged at the top end of the rocker, and the rocking handle is arranged at the bottom end of the rocker. The rocker can rotate freely in the hollow rod. Due to the fact that the taper-hopper is arranged at the lower portion of the spiral rotary broom, a user is enabled to manipulate the broom to rotate to sweep dust on the ceiling, swept dust is collected in the taper hopper, and the dust is prevented from drifting away.

Shidong et al., (2013) invented an automatic cleaner for the ceiling fan provided by the utility model that can be used for automatically cleaning dust on the ceiling fan at a high attitude and avoids the trouble that a worker climbs up to a high position; the automatic 
cleaner for the ceiling fan has the advantages of convenience in use, obvious cleaning effect, cleanliness, and sanitation; the labor, the materials and the time can be saved, the indoor dust amount is reduced, simplicity of operation and low cost, and can be generally applied to families and classrooms.

\section{CEILING MOP WITH RACK AND PINION}

The base holder which acts as a fixture of the entire setup is a hollow cylindrical pipe made of PVC pipe. At the inner portion of the base holder, a movable ram with a rack machined on its surface is placed. A linear slider is connected between the base holder and movable ram, such that it makes the linear slider slide easily inside the surface of the base holder. At the base holder, a pinion gear is mounted, which gets mesh with a rack on the surface of the movable ram. At the top portion of the ram, a motor mount fixture is attached, which helps to house an electric drive. This electric drive shaft is directly coupled with a mounting which acts as a fixture for brush and vacuum pump assembly. One more electric drive is directly coupled with pinion gear and held tightly on the base holder- mounted fixture. The power to operate the drives is provided with the help of a battery which is mounted on the base frame. The key switch is connected between the electric drive and power source to control the operation by the user. when the user turns the dc motor to operate the pinion, it helps to rotate the pinion fixed with the drive shaft about its fixed axis. This rotation is converted into linear translation motion with the help of meshed rack. Thus, the ram moves linearly to adjust the height of the cleaning set up as per the user requirement. When the desired height is reached, the dc motor is switched off to lock the position of the ram. When the brush is coupled with the upper mount, the rotation of the motor makes the brush rotate about its fixed axis and which performs the scrubbing operation on the tight particles of dust that get a stick on the surface of the ceiling. Similarly, connecting the vacuum arrangement makes the turbine blade rotate about its fixed axis, thereby creating a negative pressure that removes the dust particles just by reaching the suction chamber near the dust.

\section{HARDWARE DESCRIPTION}

(a) D.C motor:

A DC motor is any of a class of rotary electrical machines that converts direct current electrical energy into mechanical energy. The most common types rely on the forces produced by magnetic fields. Nearly all types of DC motors have some internal mechanism, either electromechanical or electronic; to periodically change the direction of current flow in part of the motor. A different number of stator and armature fields as well as how they are connected provides different inherent speed/torque regulation characteristics. The speed of a DC motor can be controlled by changing the voltage applied to the armature. In any electric motor, an operation is based on simpleelectromagnetism.

(b) Battery:

A battery is a device that converts chemical energy directly to electrical energy. It consists of a number of voltaic cells; each voltaic cell consists of two half cells connected in series by a conductive electrolyte containing anions and cations. One half-cell includes electrolyte and the electrode to which anions migrate, i.e., the anode or negative electrode; the other half-cell includes electrolyte and the electrode to which cations migrate, i.e., the cathode or positive electrode. In the redox reaction that powers the battery, reduction occurs to cations at the cathode, while oxidation occurs to anions at the anode. The electrodes do not 
touch each other but they are electrically connected by the electrolyte. Some cells use two half-cells with different electrolytes. A separator between half cells allows ions to flow but prevents the mixing of the electrolytes. Each half cell has an electromotive force determined by its ability to drive electric current from the interior to the exteriorof the cell.

(c) Metal strip:

The metal strip is a narrow, thin stock. Metal strips are formed to precise thicknesses and/or width requirements. Metal strips can be designed and manipulated through a large number of processes which are grouped into categories. They are joining and assembly processes, deformation processes, material removal processes, heat-treating processes, and finishing processes. Joining and assembly processes include welding, soldering, brazing, fastening, and other processes that connect parts permanently or semipermanently to form a new entity. Deformation processes include bending, curling, punching, rolling, deep drawing, and ironing. They include machining operations, abrasive machining, and non-traditional processes utilizing lasers and electron beams. Heat treating processes include annealing, quenching, tempering, aging, homogenizing, solution treating, and precipitation hardening.

(d) Vacuum:

The Vacuum cleaners use an electric motor that spins a fan, sucking in air and any small particles caught up in it - and pushing it out the other side, into a bag or a canister, to create the negative pressure. You might think then that after a few seconds it would stop working since you can only force so much air into a confined space. To solve this, the vacuum has an exhaust port that vents the air out the other side, allowing the motor to continue functioning normally. Vacuum is a set of normative guidance principles for achieving training and test dataset quality for structured datasets in data science and machine learning. The garbage-in, garbage-out principle motivates a solution to the problem of data quality but does not offer a specific solution. Unlike the majority of the ad-hoc data quality assessment metrics often used by practitioners, Vacuum specifies qualitative principles for data quality management and serves as a basis for defining more detailed quantitative metrics of data quality.

(e) Nylon brush head:

Nylon is one of the most commonly used polymers in brushes. The stiffness of a nylon brush is directly related to bristle diameter, length, and grade. Some of the many advantages of nylon include low to moderate water absorption, and excellent fatigue life, and the ability to work in both dry and wet environments. For industries such as semiconductor, aerospace, medical, food processing, automotive, commercial, industrial, textiles, and electronics, nylon brushes are vital components in 
many processes due to extensive application-specific configurations. There are also a variety of colors of nylon filament, which is available in its natural milky clear color, or in dyed colors such as black oryellow. As the most widely used filament in brushes, nylon is used in brushes including cleaning brushes, power brushes, spiral brushes, strip brushes, and artist brushes. Cleaning brushes such as bottle brushes are a vital component of industrial manufacturing processes that involve cleaning and can come in various sizes, depending on the application. Power brushes such as circular-shaped wheelbrushes are used to deburr parts during the initial phases of the surface finishing process. Spiral brushes mainly refer to twisted-in wire brushes, although they may also refer to several other circular or cylindricaltype brushes used in commercial and industrial manufacturing.

\section{(f) Rack and Pinion:}

A rack and pinion is a kind of straight actuator that includes a round gear drawing in direct stuff, which works to make an interpretation of rotational movement into straight movement. Driving the pinion into pivot makes the rack be driven directly. Driving the rack directly will make the pinion be crash into a revolution. The rack conveys the full heap of the actuator straightforwardly thus the driving pinion is typically little, so the stuff proportion diminishes the force required. This power, accordingly force, may, in any case, be significant thus it is basic for there to be a decrease gear preceding this by either a stuff or worm gear decrease. Rack gears have a higher proportion, in this manner requiring a more noteworthy driving force than screw actuators. While thinking about a rack and pinion for direct movement interpretation, the round pitch is one way that you can acquire a fixed travel distance without utilizing complex computations to decide the number of pivots required on the pinion to travel a specific distance. Another benefit for utilizing round contribute rather than module these applications is the thought of reaction in the framework. While going one way, the pinion has a consistent commitment with the stuff rack.

\section{D DESIGN}

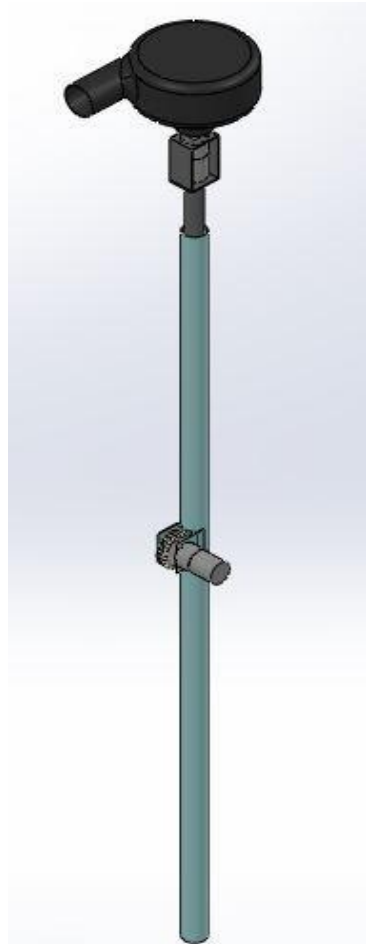

Fig.1. Vacuum Head

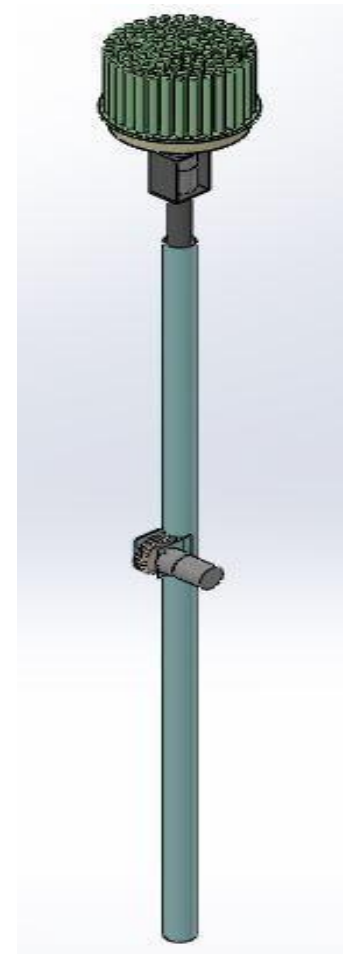

Fig.2. Rotating Brush Head 


\section{D MODEL}

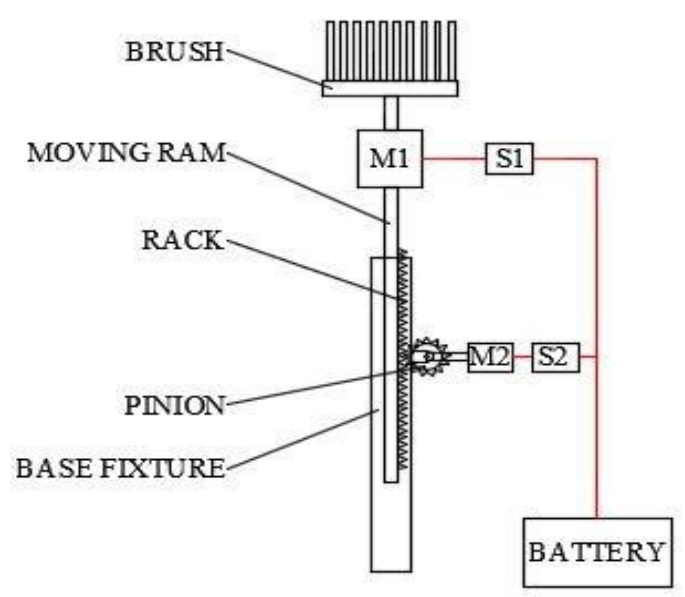

M1,2 - DC MOTORS

S1- KEY SWITCH

S2- TWO WAY SWITCH

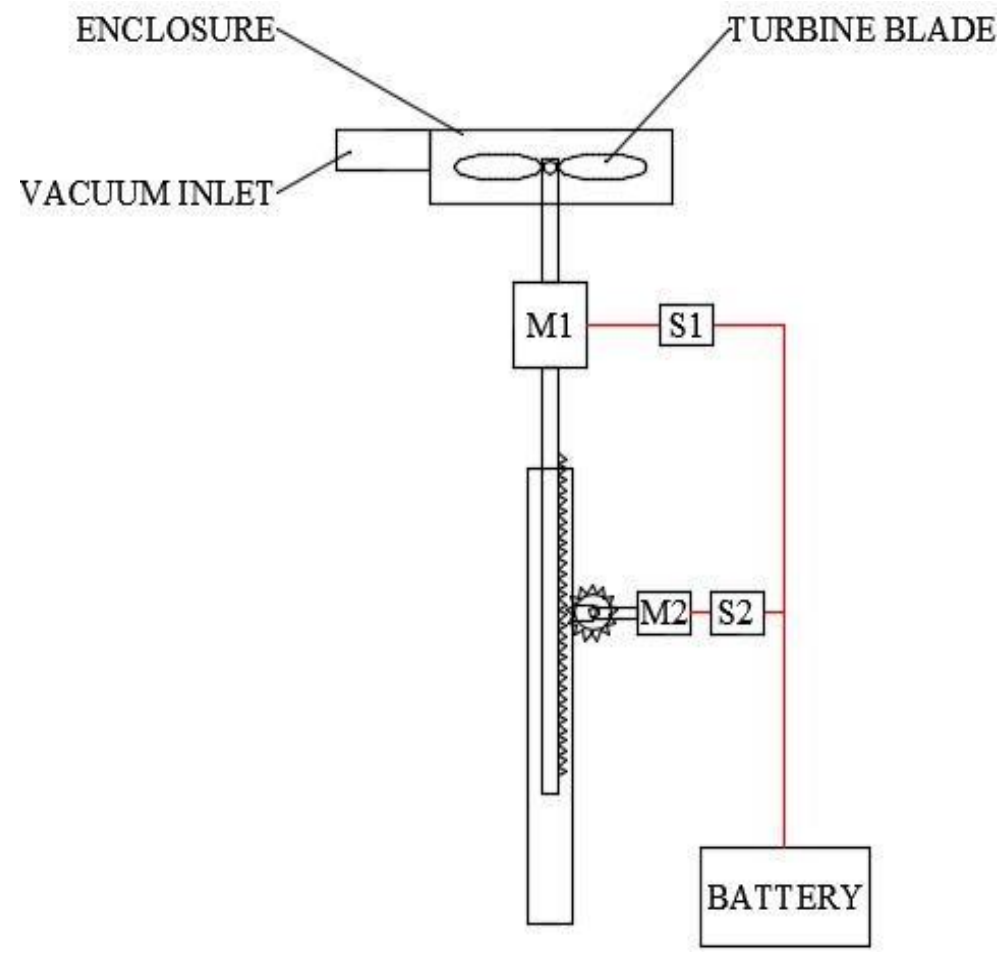

Fig.3. 2D Diagram of Rotatingand vacuum head 


\section{CONCLUSION}

In this phase-1 project, we have designed a 3D model using design software. We are using PVC tube for the outer stick so the weight of the instrument is reduced compared to the metal stick and with the help of changeable heads, the cleaning process is faster. The rack and pinion mechanism helps the stick to adjust its height based on the user's requirement.

\section{REFERENCES}

Agustin.H.C, "Device for removing cobwebs" (US5682636A), United States Patent Office, 1997.

Baines.P.J, Mills.J.K, "Feedback Linearized Joint Torque Control of a Geared, DC Motor Driven Industrial Robot", The International Journal of Robotics Research: 17(2), pp. 169192, 1998.

Bilger.D, Rifkin.A.S, "Upright Vacuum Cleaning Apparatus" (NZ631579A), New Zealand, 2013.

Bin Jasni.M.F, "Design and Fabrication of Ceiling Fan Blades Cleaner", Diploma Project in Mechanical Engineering, Universiti Malaysia Pahang, 2008.

Brendt.D, “Valve-regulated lead-acid batteries", Journal of Power Sources: 100, pp. 29-46, 2001.

Chen.Z, Fuentes-Aznar.A, Zeng.M, "Geometric Design, Meshing Simulation, and Stress Analysis of Pure Rolling Rack and Pinion Mechanisms, Journal of Mechanical Design: 142(3), 2019.

Chiasson.J, Mehta.S, "Nonlinear control of a series DC motor: theory and experiment", IEEE Transactions on Industrial Electronics: 45(1), pp. 134-141, 1998.

Dahl.I, Nilsen.S.K, Schneider.T" Cleaning methods, their effectiveness and airborne dust generation", Building and Environment: 29(3), pp. 369-372. 1994.

Dhanaseely A.J, Karmegan.C, Kumar. S.A, "Autonomous Cobweb Cleaner", Journal of Physics: Conference Series: 1717, 2021.

Dusuki.F. B, "Design and Development of Innovative Ceiling Fan Cleaner", Bachelor of EngineeringTechnology Thesis, Universiti Malaysia Pahang, 2019.

Frankovsky.P, Kenderova.M, Virgala.I, "Friction Effect Analysis of a DC Motor”, American Journalof Mechanical Engineering: 1(1), pp. 1-5, 2013

Furnemont.R, Lefeber.D, Mathijssen.G, Vanderborght.B, Verstraten.T “ Modeling and Design of geared DC motors for energy efficiency: Comparison between theory and experiments", Mechatronics: 39, pp. 198-213, 2015.

Galler.D, Kusko.A, "Control Means for Minimization of Losses in AC and DC Motor Drives”, IEEETransactions on Industry Applications: IA-19(4), pp. 561-570, 1983. 
Hardy M.T, Laithwaite E.R., "Rack-and-pinion motors: hybrid of linear and rotary machines,Proceedings of the Institution of Electrical Engineers: 117(6), pp. 1105-1112. 1970.

He Shidong “Automatic Cleaner for ceiling fan” (CN203023147U), China, 2013.

Kaneko.K, Komoriya.K, Ohnishi.K, et al., "Accurate torque control for a geared DC motor based on an acceleration controller", Proceedings of the 1992 International Conference on Industrial Electronics, Control, Instrumentation, and Automation: 1, pp. 395-400, 1992.

Liu Fangyi "Roof ceiling cleaning device”(CN101987004A), China, 2009. 
Liu Zichen "Ceiling Sweeper” (CN203107009U), China, 2013.

Louis.F, “Feather Duster” (US3162880A), United States Patent Office, 1964.Luna “Ceiling

Dust Collection Brush" (CN204839388U), China, 2015.

Ma Yuxiong “Ceiling electric cleaning ware” (CN205006820U), China, 2015.

Oslon.D, “Accelerated Tests of Wiper Motor Retainers Using CAE Durability and ReliabilityTechniques”, SAE Technical Paper 2004-01-1644, 2004.

Potter.M. F, "Eliminating Spiders around homes and Buildings", University of Kentucky, 2018.

Reiner.M, "The Centripetal-pump effect in a vacuum pump", Proceedings of the Royal Society,Mathematical, physical and Engineering Sciences:247 (1249), 1958.

Sone.Y, Sugimoto.H, "Vacuum Pump without a Moving Part and its Performance”, AIP ConferenceProceedings: 663, 2003.

Tang Jianfeng “Multifunctional Sweeper” (CN202723767U), China, 2012.Zhang Shede “Novel Broom” (CN201939279U), China, 2010.

Zhao Feng "Ceiling Dust Cleaning Device” (CN103126631A), China, 2011.Zhao Xianfeng

“Ceiling Cleaner” (CN203776855U), China, 2014. 